\title{
POWER LAW NOISE IDENTIFICATION USING THE LAG 1 AUTOCORRELATION
}

\author{
W.J. Riley* and C.A. Greenhall ${ }^{\dagger}$ \\ * Symmetricom, Inc., 34 Tozer Road, Beverly, MA 01915 USA, wriley@symmetricom.com \\ ${ }^{\dagger}$ Jet Propulsion Laboratory, 4800 Oak Grove Drive, Pasadena, CA 91109 USA, Charles.Greenhall@jpl.nasa.gov
}

Keywords: Power Law Noise, Autocorrelation, Frequency Stability Analysis.

\begin{abstract}
This paper describes a new method for power law noise identification, based on the lag 1 autocorrelation function, that can determine the dominant noise type for all common noise processes, from phase or frequency data, for all averaging factors, in a consistent and analytic manner.

\section{Background}

It is often necessary to identify the dominant power law noise process (WPM, FPM, WFM, FFM, RWFM, FWFM or RRFM) of the spectral density of the fractional frequency fluctuations, $S_{\mathrm{y}}(f)=h_{\alpha} f^{\alpha}(\alpha=2$ to -4$)$, to perform a frequency stability analysis. For example, knowledge of the noise type is necessary to determine the equivalent number of chi-squared degrees of freedom (edf) for setting confidence intervals and error bars, and it is essential to know the dominant noise type to correct for bias in the newer Total and Thêol variances. While the noise type may be known a priori or estimated manually, it is desirable to have an analytic method for power law noise identification that can be used automatically as part of a stability analysis algorithm.
\end{abstract}

\section{Prior Art}

There is little literature on the subject of power-law noise identification. The most common method for power law noise identification is simply to observe the slope of a loglog plot of the Allan or modified Allan deviation versus averaging time, either manually or by fitting a line to it. This obviously requires at least two stability points. During a stability calculation, it is desirable (or necessary) to automatically identify the power law noise type at each point, particularly if bias corrections and/or error bars must be applied. Previous methods for power law noise identification [1], based on the Barnes $B_{1}$ and $R(n)$ bias ratios [2], have been ad hoc, have not used a consistent methodology for all noise types, and have not handled all cases (e.g. resolving white and flicker PM at unity averaging factor).

\section{The Autocorrelation Function}

The autocorrelation function (ACF) is a fundamental way to describe a time series by multiplying it by a delayed version of itself, thereby showing the degree by which its value at one time is similar to its value at a certain later time. More specifically, the autocorrelation at lag $\mathrm{k}$ is defined as

$$
\rho_{k}=\frac{E\left[\left(z_{t}-\mu\right)\left(z_{t+k}-\mu\right)\right]}{\sigma_{z}^{2}}
$$

where $z_{t}$ is the time series, $\mu$ is its mean value, $\sigma_{z}^{2}$ is its variance, and $E$ denotes the expected value. The autocorrelation is usually estimated by the expression

$$
r_{k}=\frac{\frac{1}{N} \sum_{t=1}^{N-k}\left(z_{t}-\bar{z}\right)\left(z_{t+k}-\bar{z}\right)}{\frac{1}{N} \sum_{t=1}^{N}\left(z_{t}-\bar{z}\right)^{2}}
$$

where $\bar{z}$ is the mean value of the time series and $N$ is the number of data points [3].

\section{The Lag 1 Autocorrelation}

The lag 1 autocorrelation is simply the value of $r_{1}$ as given by the expression above. For frequency data, the lag 1 autocorrelation is able to easily identify white and flicker PM noise, and white (uncorrelated) FM noise, for which the expected values are $-1 / 2, \quad-1 / 3$ and zero respectively. The more divergent noises have positive $r_{1}$ values that depend on the number of samples, and tend to be larger (approaching 1). For those more divergent noises, the data are differenced until they become stationary, and the same criteria as for WPM, FPM and WFM are then used, corrected for the differencing. The results can be rounded to determine the dominant noise type or used directly to estimate the noise mixture.

\section{Noise Identification Using $r_{1}$}

An effective method for identifying power law noises using the lag 1 autocorrelation is based on the properties of discrete-time fractionally integrated noises having spectral 
densities of the form $(2 \sin \pi f)^{-2 \delta}$. For $\delta<1 / 2$, the process is stationary and has a lag 1 autocorrelation equal to $\rho_{1}=\delta$ / (1- $\delta)$ [4], and the noise type can therefore be estimated from $\delta=r_{1} /\left(1+r_{1}\right)$. For frequency data, white PM noise has $\rho_{1}=-1 / 2$, flicker PM noise has $\rho_{1}=-1 / 3$, and white FM noise has $\rho_{1}=0$. For the more divergent noises, first differences of the data are taken until a stationary process is obtained as determined by the criterion $\delta<0.25$. The noise identification method therefore uses $\mathrm{p}=$-round $(2 \delta)$ $-2 \mathrm{~d}$, where round $(2 \delta)$ is $2 \delta$ rounded to the nearest integer and $\mathrm{d}$ is the number of times that the data is differenced to bring $\delta$ down to $<0.25$. If $z$ is a $\tau$-average of frequency data $y(t)$, then $\alpha=p$; if $z$ is a $\tau$-sample of phase data $x(t)$, then $\alpha=p+2$, where $\alpha$ is the usual power law exponent $f^{\alpha}$, thereby determining the noise type at that averaging time. The properties of this power law noise identification method are summarized in Table 2. It has excellent discrimination for all common power law noises for both phase and frequency data, including difficult cases with mixed noises.

\section{Noise ID Algorithm}

The basic lag 1 autocorrelation power law noise identification algorithm is quite simple. The inputs are a vector $z_{1}, \ldots, z_{\mathrm{N}}$ of phase or frequency data, the minimum order of differencing dmin (default $=0)$, and the maximum order of differencing dmax. The output is $p$, an estimate of the $\alpha$ of the dominant power law noise type, and (optionally) the value of $d$.

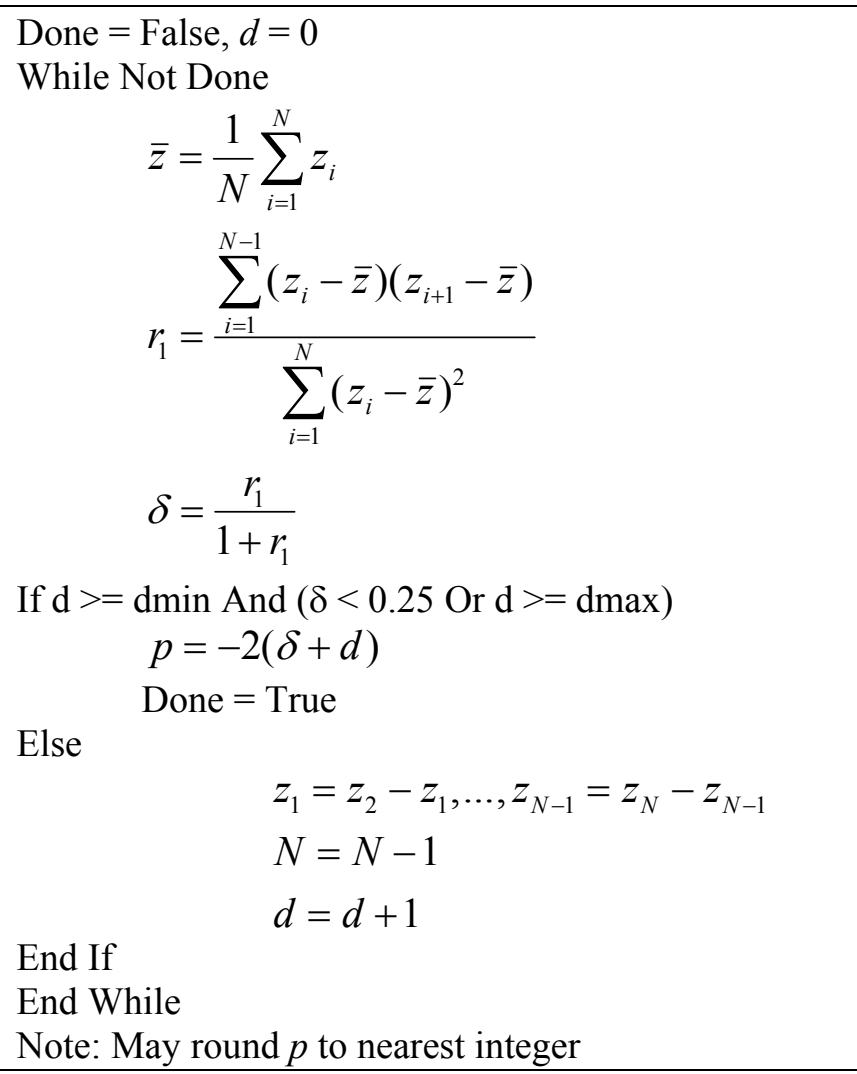

The input data should be for the particular averaging time, $\tau$, of interest, and it may therefore be necessary to decimate the phase data or average the frequency by the appropriate averaging factor before applying the noise identification algorithm. The dmax parameter should be set to 2 or 3 for an Allan or Hadamard (2 or 3-sample) variance analysis respectively. The alpha result is equal to $p+2$ or $p$ for phase or frequency data respectively, and may be rounded to an integer (although the fractional part is useful for estimated mixed noises). The algorithm is fast, requiring only the calculation of one autocorrelation value and $1^{\text {st }}$ differences for several times. It is independent of any particular variance.

\section{Results}

The lag 1 autocorrelation method yields good results, consistently identifying pure power noise for $\alpha=2$ to -4 for sample sizes of about 30 or more, and generally identifying the dominant type of mixed noises when it is at least $10 \%$ larger than the others. For a mixture of adjacent noises, the fractional result provides an indication of their ratio. For those reasons, and because it can handle all averaging factors, the new lag 1 autocorrelation method has replaced the $B_{1} / R(n)$ bias ratio method in Version 1.42a and higher of the Stable32 program [5].

\section{Examples}

Examples of the lag 1 autocorrelation method for power law noise identification are shown in Figures 1 and 2 for 100 sets of 1024 points of pure and mixed simulated noise, as generated by the Kasdin-Walter method [6], with approximately equal Allan variances $(\tau=1)$ summed for the mixed noises. Figure 4 shows a composite plot of overlapping Allan deviation and lag 1 ACF noise type for a pair of SAO VLG11B hydrogen masers. The noise type varies from white/flicker PM at short averaging times to more divergent random walk/flicker walk FM at longer averaging times. Figure 5 shows a composite plot of overlapping Allan deviation and Lag 1 ACF noise type for a Symmetricom Cs-III high performance laboratory cesium frequency standard vs. a Symmetricom MHM 2010 hydrogen maser. As is typical for such devices, it displays white FM noise out to an averaging time of several days before reaching a "flicker floor". Figure 6 shows a composite plot of overlapping Allan deviation and Lag 1 ACF noise type for a Symmetricom Model 8130 militarized rubidium frequency standard, again vs. a Symmetricom MHM 2010 hydrogen maser. It displays white FM noise before reaching a region of flicker and random walk FM noise at an averaging time of about $10^{4}$ seconds.

\section{Limitations}

Before analysis, the data should be preprocessed to remove outliers, discontinuities, and deterministic components. Simulations using 100 sets of white FM noise of various 
sample sizes have shown that acceptable results can be obtained from the lag 1 autocorrelation noise identification method for $\mathrm{N} \geq 32$, where $\mathrm{N}$ is the number of data points, as shown in the Table 1 . The table shows the percentage of estimates that differ from the expected $\alpha$ value of 0 by more than a half-integer noise type. This percentage includes whatever error is due to the simulated noise data itself.

\begin{tabular}{|c|c|c|}
\hline $\mathrm{N}$ & $\%$ & $\alpha$ Range \\
\hline 32 & 16 & -0.4 to +1.4 \\
\hline 64 & 6 & -0.4 to +0.7 \\
\hline 128 & 1 & -0.3 to +0.5 \\
\hline 256 & 0 & -0.2 to +0.3 \\
\hline 512 & 0 & -0.2 to +0.2 \\
\hline 1024 & 0 & -0.1 to +0.2 \\
\hline
\end{tabular}

Table 1: Percent Incorrect Power Law Noise Estimates vs. Sample Size

It would be interesting to devise an improved lag 1 autocorrelation noise identification method (perhaps based on reflected data like the Total variance) that could work down to smaller sample sizes. The algorithm tends to produce jumps in the estimated alpha for mixed noises when the differencing factor, $d$, changes (although the alpha value when rounded to an integer is still consistent). As shown in Figures 3, this can be avoided by using the same $d$ for the entire range of averaging times, at the expense of higher variability when a lower d would have been sufficient. Further research is encouraged to develop even better ways to identify power law noise.

\section{Conclusion}

This paper has described a method for power law noise identification based on the lag 1 autocorrelation function. It is a fast and effective way to support the setting of confidence intervals and to apply bias corrections during a frequency stability analysis.

\section{References}

1. D. Howe, R. Beard, C. Greenhall, F. Vernotte and B. Riley, "A Total Estimator of the Hadamard Function Used by GPS Operations", Proc. $32^{\text {nd }}$ Precise Time and Time Interval (PTTI) Systems and Applications Meeting, November 2000, pp. 255-268.

2. J.A. Barnes, "Tables of Bias Functions, B1 and B2, for Variances Based on Finite Samples of Processes with Power Law Spectral Densities", NBS Technical Note 375, Jan. 1969.

3. G. Box and G. Jenkins, Time Series Analysis, Forecasting and Control, Chapter 2, Holden-Day, Oakland, California, 1976, ISBN 0-8162-1104-3.

4. P. Brockwell and R. Davis, Time Series: Theory and Methods, $2^{\text {nd }}$ Edition, Eq. (13.2.9), Springer-Verlag, New York, 1991.

5. Stable32, Software for Frequency Stability Analysis, Hamilton Technical Services, 195 Woodbury Street, South Hamilton, MA 01982 USA, http://www.wriley.com.

6. N.J. Kasdin and T. Walter, "Discrete Simulation of Power Law Noise", Proc. 1992 IEEE Frequency Control Symposium, pp. 274-283, May 1992.

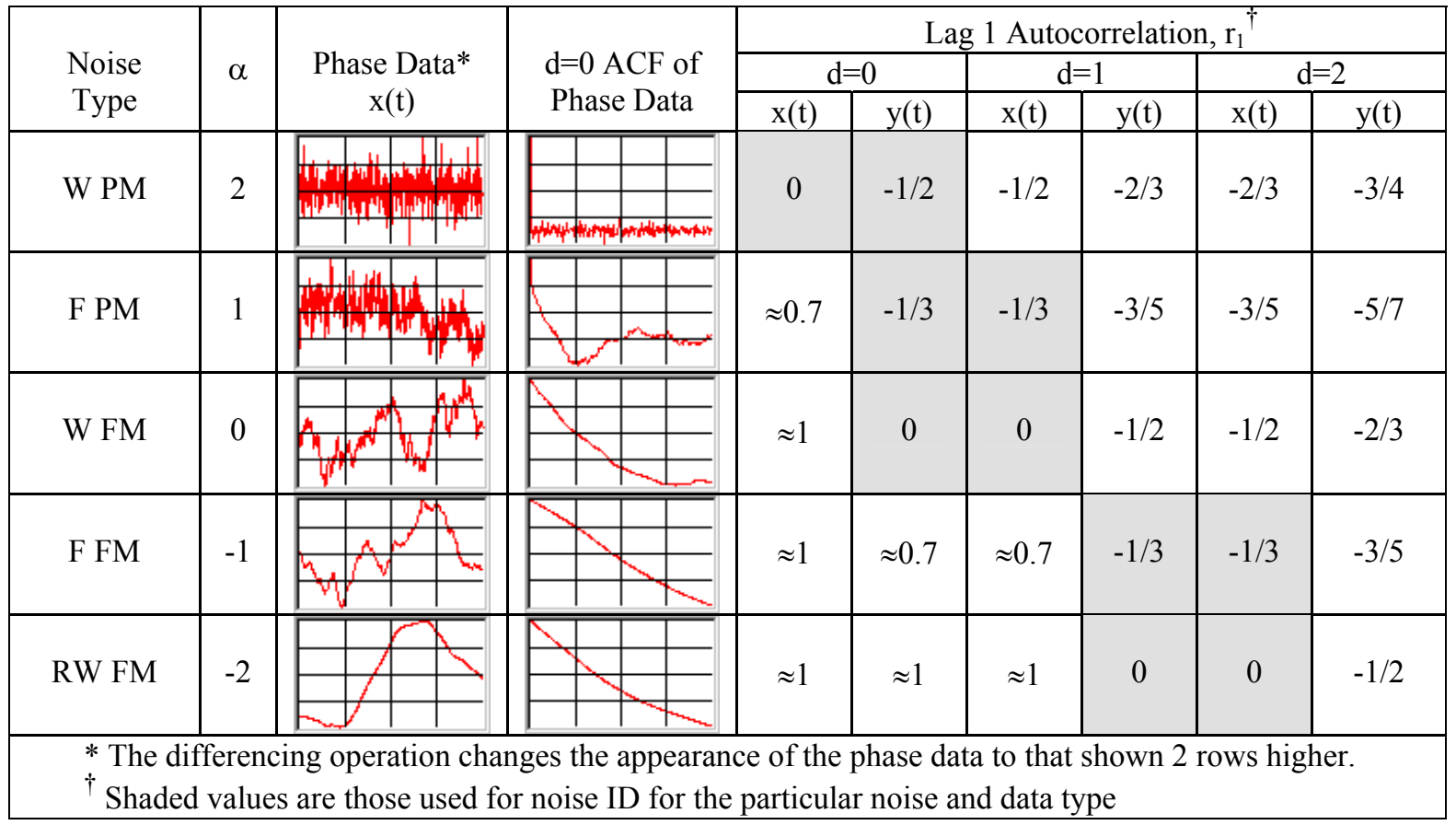

Table 2: Lag 1 Autocorrelation for Various Power Law Noises and Differences 
Lag 1 Autocorrelation Estimated Pure Integer Power Law Noise Type ( $\mathrm{N}=1024)$

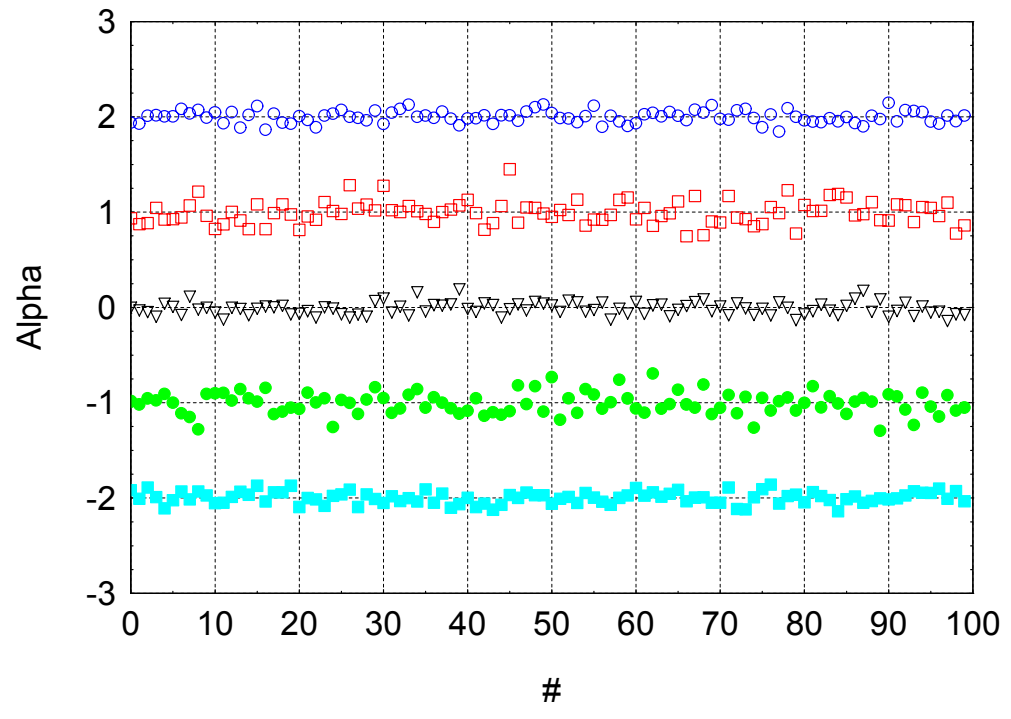

Wax $=+2.147$

W PM, $\alpha=+2$

Avg $=+1.9998$

$\operatorname{Min}=+1.844$

F PM, $\alpha=+1$

$\operatorname{Max}=+1.449$

Avg $=+0.9957$

$\operatorname{Min}=+0.745$

W FM, $\alpha=0$

$\operatorname{Max}=+0.204$

Avg $=-0.0004$

Min $=-0.129$

F FM, $\alpha=-1$

Max $=-0.694$

Avg $=-1.0045$

Min $=-1.295$

RW FM, $\alpha=-2$

Ma $\mathrm{x}=-1.860$

Avg $=-1.9960$

$\operatorname{Min}=-2.138$

Figure 1: Examples of Lag 1 Autocorrelation Noise ID for Pure Integer Simulated Power Law Noises

Lag 1 Autocorrelation Estimated Mixed Integer Power Law Noise Type (N=1024)

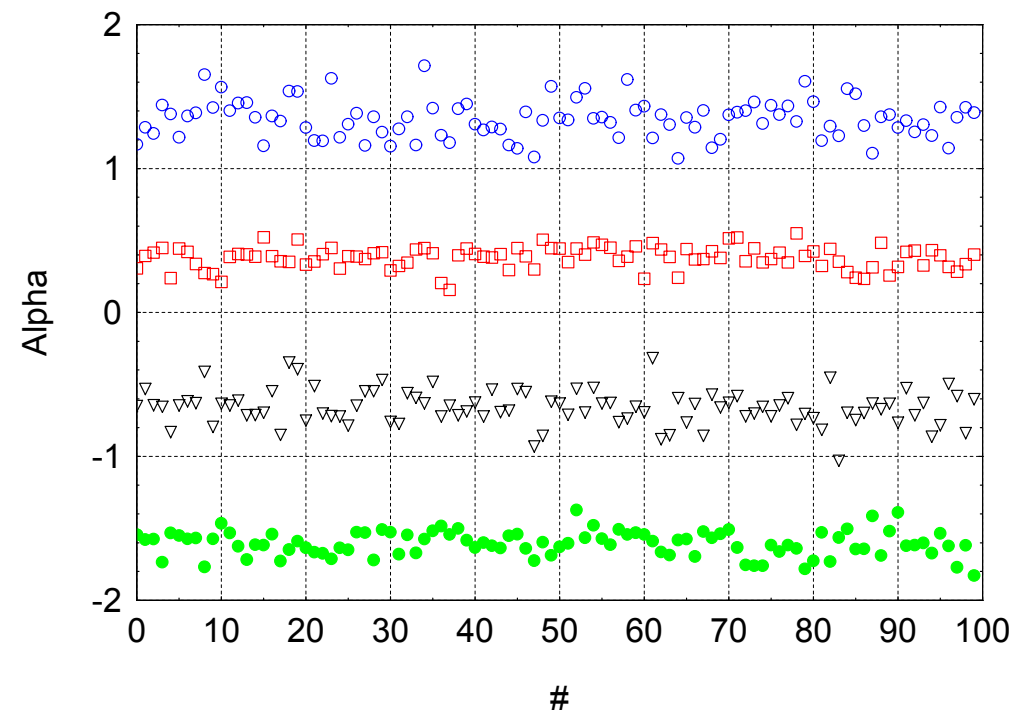

W PM \& F PM, $\alpha=+1.5$

$\operatorname{Max}=+1.714$

Avg $=+1.3431(1.359)$

$\operatorname{Min}=+1.071$

F PM \& W FM, $\alpha=+0.5$

$\operatorname{Max}=+0.549$

Avg $=+0.3802(0.373)$

Min $=+0.157$

W FM \& F FM, $\alpha=-0.5$

$\operatorname{Max}=-0.308$

Avg $=-0.6534(-0.656)$

Min $=-1.021$

F FM \& RW FM, $\alpha=-1.5$

$\operatorname{Max}=-1.373$

Avg $=-1.6041(-1.610)$

Min $=-1.828$

Note: Theoretical values shown in ()

Figure 2: Examples of Lag 1 Autocorrelation Noise ID for 50\% Mixture of Adjacent Integer Power Law Noises

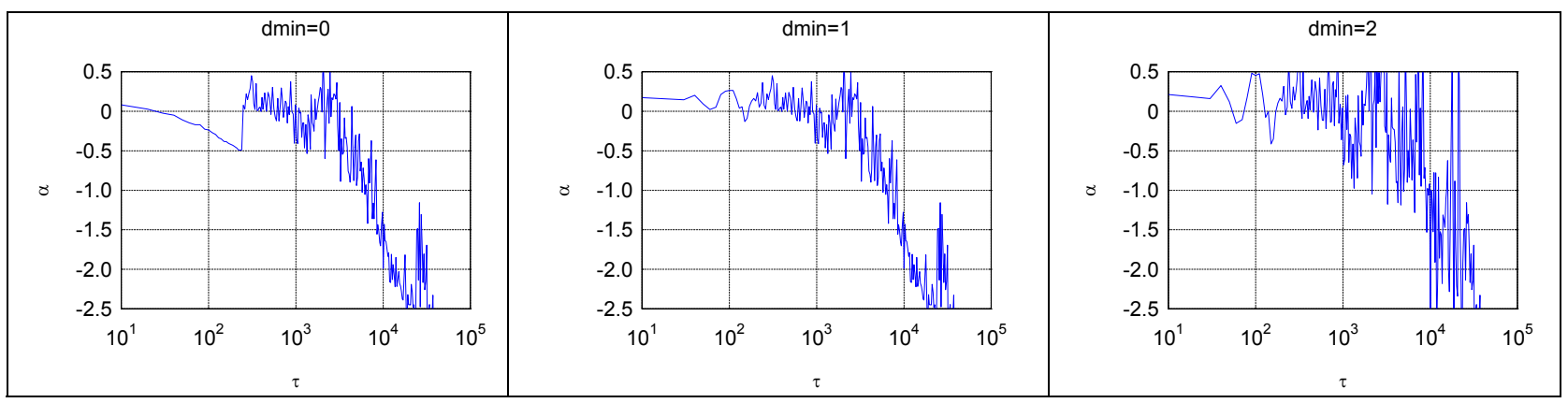

Figure 3: Noise Analysis of the Rubidium Frequency Standard of Figure 6 for dmin $=0,1$ and 2 


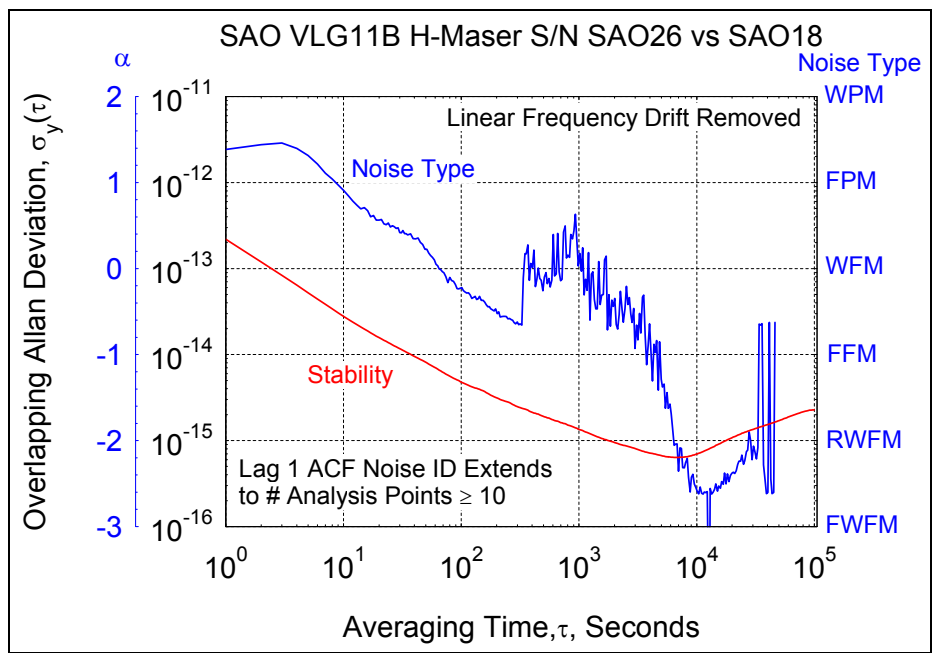

Figure 4: Frequency Stability and Noise Analysis of Two Hydrogen Masers

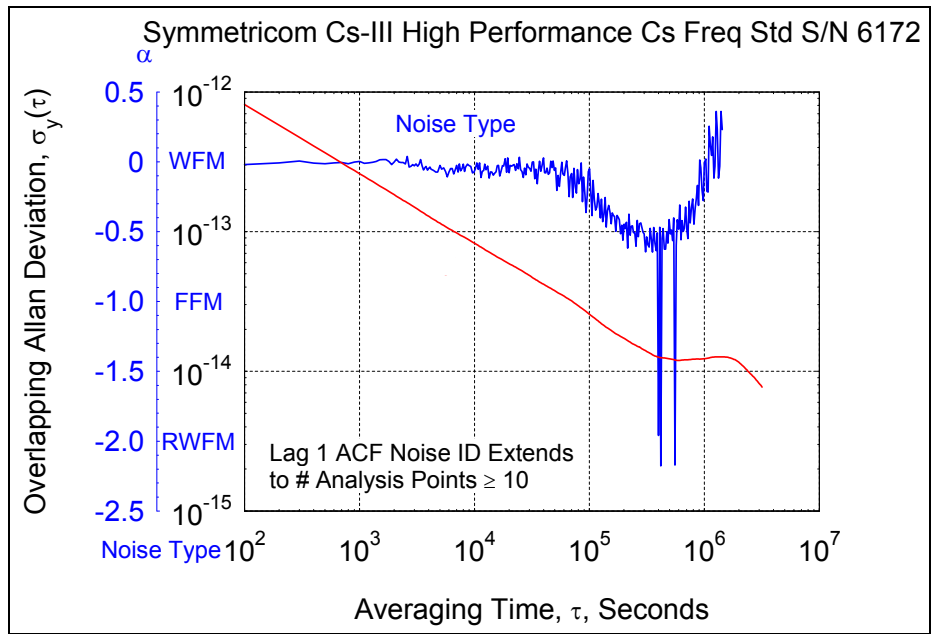

Figure 5: Frequency Stability and Noise Analysis of a Cesium Frequency Standard

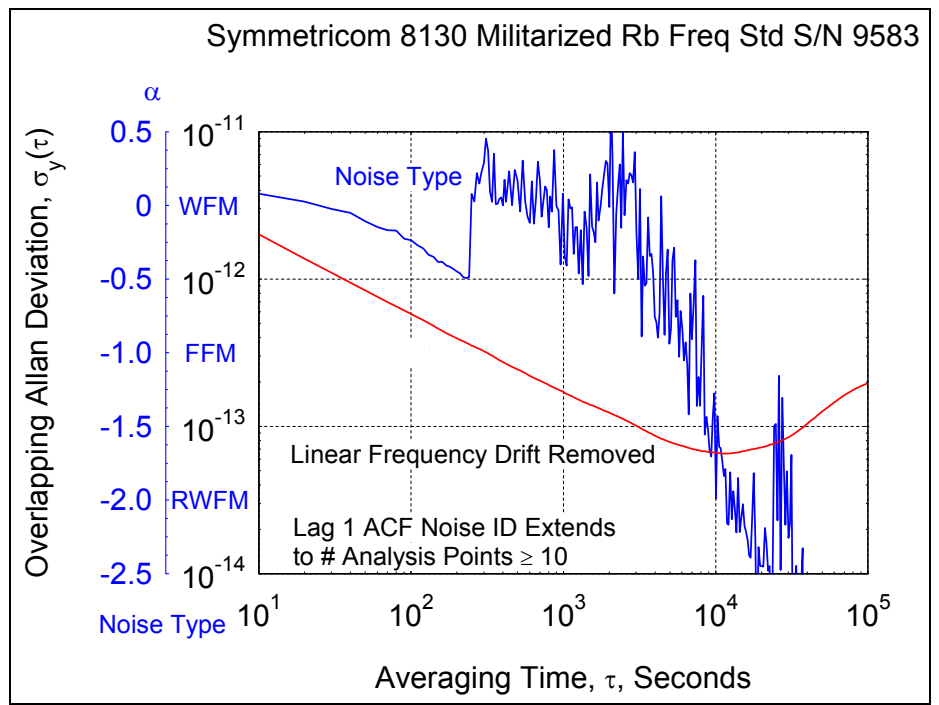

Figure 6: Frequency Stability and Noise Analysis of a Rubidium Frequency Standard 